\title{
Scatter Characterization and Correction for Simultaneous Multiple Small-Animal PET Imaging
}

\author{
Rameshwar Prasad, ${ }^{1}$ Habib Zaidi ${ }^{1,2,3}$ \\ ${ }^{1}$ Division of Nuclear Medicine and Molecular Imaging, Geneva University Hospital, 1211, Geneva, Switzerland \\ ${ }^{2}$ Geneva Neuroscience Center, Geneva University, 1211, Geneva, Switzerland \\ ${ }^{3}$ Department of Nuclear Medicine and Molecular Imaging, University of Groningen, University Medical Center Groningen, 9700 RB, \\ Groningen, Netherlands
}

\begin{abstract}
Purpose: The rapid growth and usage of small-animal positron emission tomography (PET) in molecular imaging research has led to increased demand on PET scanner's time. One potential solution to increase throughput is to scan multiple rodents simultaneously. However, this is achieved at the expense of deterioration of image quality and loss of quantitative accuracy owing to enhanced effects of photon attenuation and Compton scattering. The purpose of this work is, first, to characterize the magnitude and spatial distribution of the scatter component in small-animal PET imaging when scanning single and multiple rodents simultaneously and, second, to assess the relevance and evaluate the performance of scatter correction under similar conditions.

Methods: The LabPETTM -8 scanner was modelled as realistically as possible using Geant4 Application for Tomographic Emission Monte Carlo simulation platform. Monte Carlo simulations allow the separation of unscattered and scattered coincidences and as such enable detailed assessment of the scatter component and its origin. Simple shape-based and more realistic voxel-based phantoms were used to simulate single and multiple PET imaging studies. The modelled scatter component using the single-scatter simulation technique was compared to Monte Carlo simulation results. PET images were also corrected for attenuation and the combined effect of attenuation and scatter on single and multiple small-animal PET imaging evaluated in terms of image quality and quantitative accuracy.

Results: A good agreement was observed between calculated and Monte Carlo simulated scatter profiles for single- and multiple-subject imaging. In the LabPETTM_8 scanner, the detector covering material (kovar) contributed the maximum amount of scatter events while the scatter contribution due to lead shielding is negligible. The out-of field-of-view (FOV) scatter fraction (SF) is $1.70,0.76$, and $0.11 \%$ for lower energy thresholds of 250,350 , and $400 \mathrm{keV}$, respectively. The increase in SF ranged between 25 and $64 \%$ when imaging multiple subjects (three to five) of different size simultaneously in comparison to imaging a single subject. The spill-over ratio (SOR) increases with increasing the number of subjects in the FOV. Scatter correction improved the SOR for both water and air cold compartments of single and multiple imaging studies. The recovery coefficients for different body parts of the mouse whole-body and rat whole-body anatomical models were improved for multiple imaging studies following scatter correction.
\end{abstract}

Correspondence to: Habib Zaidi; e-mail: habib.zaidi@hcuge.ch 
Conclusions: The magnitude and spatial distribution of the scatter component in small-animal PET imaging of single and multiple subjects simultaneously were characterized, and its impact was evaluated in different situations. Scatter correction improves PET image quality and quantitative accuracy for single rat and simultaneous multiple mice and rat imaging studies, whereas its impact is insignificant in single mouse imaging.

Key words: PET, Small animals, Scatter, Simultaneous multiple imaging, Monte Carlo simulation

\section{Introduction}

$\mathrm{I}^{\mathrm{n}}$ recent years, small-animal positron emission tomography (PET) has gained popularity and established its utility in the biomedical research arena owing to its high sensitivity and capability to provide quantitative measurements of biochemical and molecular processes in vivo [1, 2]. For optimal image quality and accurate quantification in PET imaging, object-specific correction of background (randoms) and physical degrading factors such as photon attenuation, Compton scatter, partial volume effects and motion have to be compensated for prior to reconstruction or incorporated within statistical iterative image reconstruction techniques $[3,4]$. However, little has been published on the assessment of the quantitative capability of small-animal PET studies [5-7].

Attenuation is the loss of true events due to scatter and absorption which impacts the measured data and results in inaccurate quantification of reconstructed PET images, whereas scatter degrades image contrast and affects both relative and absolute PET quantification. The scatter magnitude and spatial distribution depend mainly on the size and density of the object and scanner-related parameters such as design geometry, detector energy resolution and acquisition energy window [8]. In clinical PET imaging, it is well established that scatter correction is essential for accurate quantification of tracer uptake since the scatter fraction (SF) varies between 30 and $35 \%$ in brain imaging and 50 and $60 \%$ in whole-body imaging in three-dimensional acquisition mode $[9,10]$. On the other hand, when imaging small subjects such as rodents, scatter is assumed to be a minor factor due to the small size of the animals. The origin of scatter for small-animal imaging has not been well characterized, but has been proposed to stem mainly from the gantry and environment rather than the animal itself [11]. This opinion is supported by the fact that scatter correction usually does not involve correcting for scatter in the detector crystal. However, annihilation photons are subject to attenuation and scatter even in small animals such as mice and rats $[12,13]$. SF estimates ranging from 5 to $21 \%$ for mice and 15 to $30 \%$ for rats have been reported [11, 14-18]. Another ${ }^{11} \mathrm{C}$-raclopride PET study reported a larger SF (25$45 \%)$ in the rat brain with an increase in the distribution volume ratio of $3.5 \%$ after scatter correction [19].
It has become a common practice nowadays to scan multiple rodents simultaneously at different radial offsets in the scanner's field-of-view (FOV) to increase the throughput in small-animal PET imaging [20-22]. However, this is achieved at the expense of deterioration of image quality and loss of quantitative accuracy due to combined effects of photon attenuation and Compton scatter [23]. The lack of literature reporting on the magnitude, spatial distribution of the scatter component and effect of scatter correction on image quality and quantitative accuracy when imaging multiple rodents simultaneously motivated this study.

The purpose of this work is, first, to characterize the magnitude and spatial distribution of the scatter component in small-animal PET imaging when scanning single and multiple rodents (mice and rats) simultaneously and, second, to assess the relevance and impact of scatter correction on image quality and quantitative accuracy under similar conditions.

\section{Materials and Methods}

\section{Small-Animal PET System}

The LabPETTM 8 is the PET subsystem of the integrated trimodality Triumph ${ }^{\mathrm{TM}} \mathrm{PET} / \mathrm{SPECT} / \mathrm{CT}$ pre-clinical imaging system (Gamma Medica, Inc., Northridge, CA). It is a state-of-art avalanche photodiode (APD)-based digital small-animal PET scanner designed with quasi-individual crystal readout along with parallel digital architecture to achieve high performance [24]. The scanner has $7.5-\mathrm{cm}$ axial and $10-\mathrm{cm}$ transaxial FOVs. Scintillators of $2 \times 2 \times 12 / 14 \mathrm{~mm}^{3}$ in size are composed of a $\mathrm{Lu}_{0.4} \mathrm{Gd}_{1.6} \mathrm{SiO}_{5}$ (LGSO) and $\mathrm{Lu}_{1.9} \mathrm{Y}_{0.1} \mathrm{SiO}_{5}$ (LYSO). These are optically coupled one after the other, forming phoswich pairs of detectors. Four phoswich detectors are enclosed in a hermetic container made of kovar (an alloy of iron-nickel-cobalt having a density of $8.359 \mathrm{~g} /$ $\mathrm{cm}^{3}$ ). The end of the axial FOV shielding is made of tungsten $\left(19.3 \mathrm{~g} / \mathrm{cm}^{3}\right.$ density, $15.75 \mathrm{~mm}$ thickness and $131 \mathrm{~mm}$ internal diameter) to minimize the detection of out-of-FOV activity. The most relevant design features of the LabPET ${ }^{\mathrm{TM}}-8$ can be found in [24], whereas the technical specifications and performance assessment of the system used in our laboratory are given elsewhere [17].

\section{Monte Carlo Simulation Studies}

Monte Carlo simulations provide the ability to generate data under controlled conditions and to discriminate between scattered and unscattered events [25]. As such, it is considered as a useful tool to 
assist in the development and evaluation of scatter correction techniques. An experimentally validated Monte Carlo simulation model of the LabPET TM-8 scanner was used in this work [8]. The LabPET ${ }^{\text {TM}}-8$ scanner is modelled as realistically as possible in terms of geometry, physics of radiation transport and signal processing using the Geant4 Application for Tomographic Emission (GATE) Monte Carlo simulation toolkit [26]. All simulations were run using a coincidence timing window of $10 \mathrm{~ns}$, an energy resolution of $25 \%$ and lower energy thresholds of 250, 350 and $425 \mathrm{keV}$, while the upper energy threshold was kept constant $(650 \mathrm{keV})$. Back-to-back $511 \mathrm{keV}$ annihilation photons were generated to decrease computational time. A total of $10^{9}$ events were generated for each simulation study, yielding count rates equivalent to those encountered in typical smallanimal PET studies. The GATE output information comprising separate prompt and scattered events was recorded in ASCII format. The SF, estimated as the ratio of scattered to total events, was then calculated. In a second step, coincidence events were binned into sinograms for further characterization of the spatial distribution of Compton scatter. Reconstructions were performed by means of the Software for Tomographic Image Reconstruction (STIR) using the iterative three-dimensional ordered subsets maximum a posteriori using the one-step late (OSMAPOSL) algorithm with four subsets and eight iterations [27].

Various simple digital phantoms and computerized anatomical models of rodents enabling to simulate simple source geometries and more realistic complex patterns of tracer distributions were used to assess the magnitude and spatial distribution of scattered events [28]. Rodent studies were simulated using typical spatial biodistribution of 2-deoxy-2-[ $\left[{ }^{18} \mathrm{~F}\right]$ fluoro-D-glucose for mice and rats $[29,30]$ considering the corresponding distribution of attenuation coefficients. The NEMA NU-04 image quality phantom [31] and more realistic digital phantoms such as mouse whole body (MOBY) and rat whole body (ROBY) [32] were used to study the scatter component for single- and multiple-subject scanning configurations. The different arrangements of phantoms adopted in this work are shown in Fig. 1. In the 'single-subject' configuration, one subject was located in the centre of the scanner's FOV. In the 'three-subject' configuration, two subjects were located on the left and right sides of the centrally located subject, whereas in the 'five-subject' configuration, five subjects were located at the left, centre, right, top and bottom positions in the scanner's FOV. The true image obtained by reconstructing the unscattered component from the GATE simulations is used as reference for comparison.

\section{Evaluation of the Scatter Component and Out-of-FOV Scatter}

The scatter component of the LabPET ${ }^{\mathrm{TM}}-8$ scanner was characterized in terms of object and detector/gantry scatter. Object scatter consists of annihilation photons which have undergone Compton scattering only in the object, whereas detector/gantry scatter represents Compton scattering in different components of the scanner other than the object under study. This includes scintillation crystals, scanner gantry, detector covering and end-of-FOV shielding. Compton-scattered events in these different components were also evaluated.

The magnitude of out-of-FOV scatter for the LabPETTM-8 scanner was evaluated using both simulated and experimental measurements. This was performed using short $(7 \mathrm{~cm}$ height and $2.5 \mathrm{~cm}$ diameter) and long (14 cm high and $2.5 \mathrm{~cm}$ in diameter) solid polyethylene phantoms. The short phantom was placed within the axial FOV of the scanner, while the long phantom was placed within the axial FOV extending outside the axial FOV at both ends. A line source ( $7 \mathrm{~cm}$ long) containing $10 \mathrm{MBq}$ of F-18 was inserted into the short phantom parallel to its long axis, whereas a line source (14 cm long) containing $20 \mathrm{MBq}$ of F-18 was used for the long phantom. The SF was calculated according to the method described in the NEMA NU-04 standards [31]. Scatter from out-ofFOV activity was calculated by subtracting the SF of the short phantom from that of the long phantom.

\section{Scatter Modelling and Correction}

Model-based scatter estimation techniques use emission and transmission images to estimate the probability of scattered events along each line of response for selected scatter points in the transmission image by calculating the line integral of the scatter photon trajectories [9]. The scatter distribution is calculated by summation of the probabilities of Compton scattering estimated for each possible scatter point in the transmission image and every detector pair. The single-scatter simulation (SSS) technique used in this work is based on the single-scatter approximation and estimates the scatter distribution using the Klein-Nishina formula [33].

The scatter distribution is calculated using the SSS technique, which is scaled to account for multiple scatter and scatter from outof-FOV activity. However, the implementation in STIR package is optimized for clinical scanners where coarse sampling is used to speed up the scatter computation, and the full scatter sinogram is constructed by interpolation [34]. The number of detectors is smaller in small-animal PET scanners compared to clinical scanners and, as such, full detector sampling was adopted. The resulting scatter sinogram obtained using two iterations was compared to Monte Carlo simulated scatter sinograms for both single- and simultaneous multiple-subject studies.

The PET data were corrected for attenuation by multiplying the attenuation correction factors obtained by forward projection of the object(s)-specific attenuation map at $511 \mathrm{keV}$. The scatter sinogram was used as additive terms in the iterative OSMAPOSL algorithm using STIR.

\section{Image Quality and Quantitative Accuracy}

The effect of scatter correction on image quality and quantitative accuracy was evaluated for single- and simultaneous multiplesubject PET imaging using various figures of merits including contrast, signal-to-noise ratio (SNR), spill-over ratio (SOR) and recovery coefficients. Regions of interest (ROIs) with diameters approximately equal to half the radius of the two cold cylinders (no radioactivity) were defined and the mean counts/ROI in each region and in the background of the NEMA image quality phantom determined for true, uncorrected, attenuation corrected only and attenuation and scatter-corrected images. SOR was calculated as the ratio of counts in the respective cold cylinder to the counts in the uniformly active region. The contrast recovery (ideal value of $100 \%$ ) was calculated according to:

$$
\text { Contrast }=\left(\frac{N_{B G}-N_{\text {cold }}}{N_{B G}}\right) 100 \%
$$



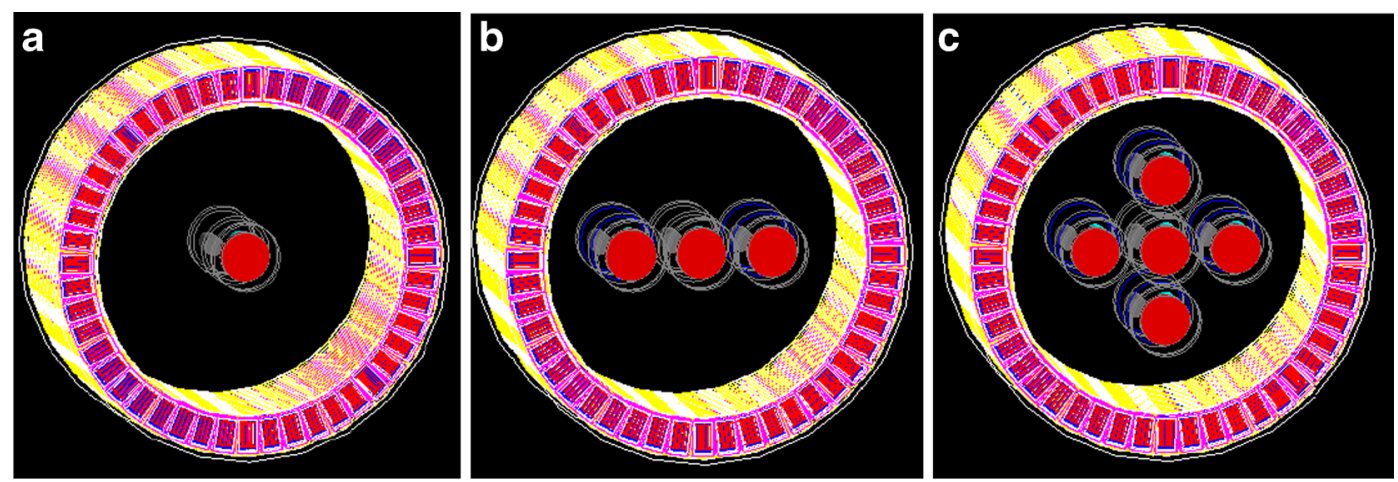

Fig. 1. Illustration of single- and multiple-subject imaging configurations used in this study showing: a single subject located centrally in the FOV, $\mathbf{b}$ three subjects in the FOV placed on the left, central and right positions and $\mathbf{c}$ five subjects in the FOV placed on the left, central, right, up and down positions.

where $N_{\mathrm{BG}}$ is the count density in the background's ROI, and $N_{\text {cold }}$ is the count density in the cold cylinder's (air or water) ROI.

The SNR was defined as the mean number of counts divided by the standard deviation of pixel intensities in a ROI defined within the background region of the NEMA image quality phantom.

$S N R=\left(\frac{\overline{N_{R O I}}}{\sigma_{R O I}}\right)$

The recovery coefficient, defined as the percentage ratio of counts in an ROI in the corrected image to those in the same ROI in the true images, was calculated according to:

Recoverycoefficient $=\left(\frac{N_{R O I}^{\text {corrected }}}{N_{R O I}^{\text {true }}}\right) \times 100 \%$

\section{Results}

\section{Modelling the Scatter Component}

The magnitude of Compton scattering originating from the various physical materials present in the LabPET ${ }^{\mathrm{TM}}-8$ scanner and the amount of out-of-FOV scatter for various energy windows are summarized in Table 1 . The maximum

Table 1. The scatter fraction due to different materials in the LabPET ${ }^{\mathrm{TM}}-8$ scanner for lower energy thresholds of 250,350 and $400 \mathrm{keV}$. The experimental out-of-FOV SF values are also shown between brackets

\begin{tabular}{llll}
\hline Materials & \multicolumn{3}{l}{ Scatter fraction (\%) } \\
\cline { 2 - 4 } & $250-$ & $\begin{array}{l}350- \\
650 \mathrm{keV}\end{array}$ & $\begin{array}{l}400- \\
650 \mathrm{keV}\end{array}$ \\
\hline Kovar & $650 \mathrm{keV}$ & 17.16 & 10.71 \\
Copper & 22.51 & 2.40 & 1.19 \\
Lead & 5.93 & 0.65 & 0.30 \\
Out-of-FOV & 1.71 & $0.76(0.81)$ & $0.11(0.12)$ \\
\hline
\end{tabular}

amount of scatter observed is due to kovar, which is used as a covering material for the detectors. The scatter contribution due to lead shielding is negligible. Both experimental and simulated out-of-FOV SFs show that out-of-FOV scatter is negligible in small-animal PET imaging using the LabPET $^{\mathrm{TM}}-8$ scanner. The effect of increasing the number of objects in scanner's FOV on the magnitude of scatter can be observed in Table 2. In the case of MOBY, the percentage increase in SF for three and five subjects is 36.4 and $63.6 \%$ in comparison to one subject, respectively. Full axial lengths of the NEMA image quality phantom and MOBY model were covered in one acquisition, whereas the ROBY model was scanned at three different axial positions corresponding to brain, thorax and pelvis regions. As expected, object SF decreases with increasing the LET.

The good agreement between experimentally measured and Monte Carlo simulated projection data (total events) for a single NEMA image quality phantom is depicted in Fig. 2 for both the uniform region and region-containing active rods. Comparisons between the scatter distributions obtained using model-based scatter estimation and Monte Carlo simulations

Table 2. Object scatter fraction estimates for single and simultaneous multiple scanning of the NEMA image quality phantom, MOBY and ROBY for lower energy thresholds of 250, 350 and $400 \mathrm{keV}$. The percentage increase in SF for three and five subjects with respect to single-subject configuration is shown between brackets

\begin{tabular}{llll}
\hline Objects & \multicolumn{2}{l}{ Scatter fraction $(\%)$} & \\
\cline { 2 - 4 } & $250-650 \mathrm{keV}$ & $350-650 \mathrm{keV}$ & $400-650 \mathrm{keV}$ \\
\hline 1 NEMA phantom & 8.10 & 5.08 & 3.01 \\
3 NEMA phantoms & $11.09(36.91)$ & $7.09(39.57)$ & $3.81(26.58)$ \\
5 NEMA phantoms & $13.11(61.85)$ & $8.13(60.04)$ & $4.03(33.89)$ \\
1 MOBY & 11.21 & 7.05 & 4.03 \\
3 MOBY & $15.19(35.50)$ & $10.03(42.27)$ & $5.01(24.32)$ \\
5 MOBY & $18.15(61.91)$ & $11.04(56.60)$ & $6.02(49.38)$ \\
1 ROBY (brain) & 12.01 & 9.00 & 6.02 \\
2 ROBY (brain) & $17.03(41.80)$ & $13.01(44.56)$ & $8.00(32.89)$ \\
1 ROBY (thorax) & 18.04 & 15.06 & 10.04 \\
2 ROBY (thorax) & $25.06(38.91)$ & $20.09(33.40)$ & $14.05(39.94)$ \\
1 ROBY (pelvis) & 20.01 & 16.07 & 11.06 \\
2 ROBY (pelvis) & $28.00(39.93)$ & $21.08(31.18)$ & $15.04(35.99)$ \\
\hline
\end{tabular}



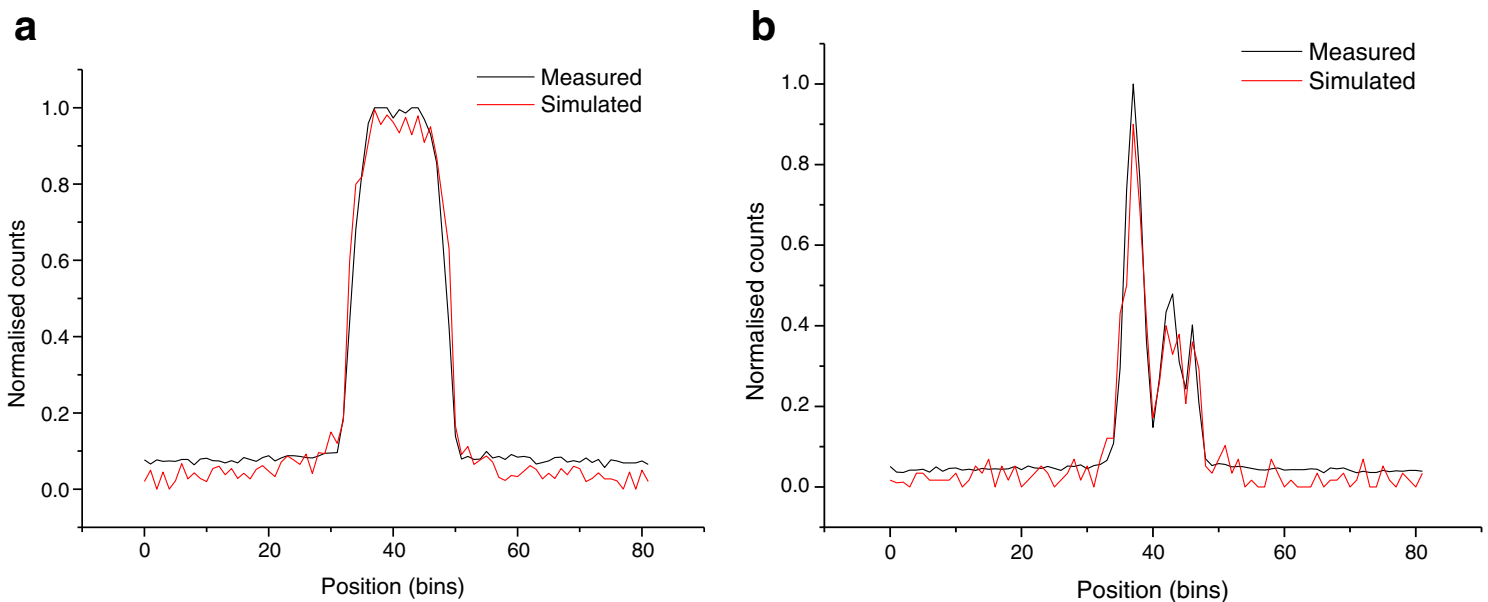

Fig. 2. Comparison of experimental and Monte Carlo simulated projection data (total events) for the single NEMA image quality phantom through $\mathbf{a}$ the uniform region and $\mathbf{b}$ the region covering the active rods.

for single and multiple scanning of the NEMA image quality phantom and ROBY are shown in Figs. 3 and 4, respectively. Overall, a good agreement was observed between model-based and Monte Carlo simulated scatter profiles. A small discrepancy between the tails of the scatter profiles can be observed, which is likely due to the fact that analytical modelling of multiple scattering does not account for the different angular distributions of the rescattered photons.

\section{Assessment of Image Quality and Quantitative Accuracy}

In the uniform compartment of the NEMA phantoms, the SNR for the five subjects is less than that of the three subject arrangement, the latter being also less than that of the single phantom configuration (data not shown). Scatter-corrected images have a poorer SNR, which can be explained by the scatter subtraction process. The SORs measured in the cold compartment of the NEMA phantom are shown in Table 3. The SOR increased in the case of multiple subjects scanning compared to single-subject scanning. Scatter correction decreases the SOR in both air- and water-filled compartments in the configurations adopted in this study. However, the effect of scatter correction was more noticeable in the air compartment than in the water compartment. The effect of attenuation and scatter correction on image contrast is demonstrated in Table 4 for different configurations of the NEMA image quality phantom. The contrast was more degraded in the multiple a
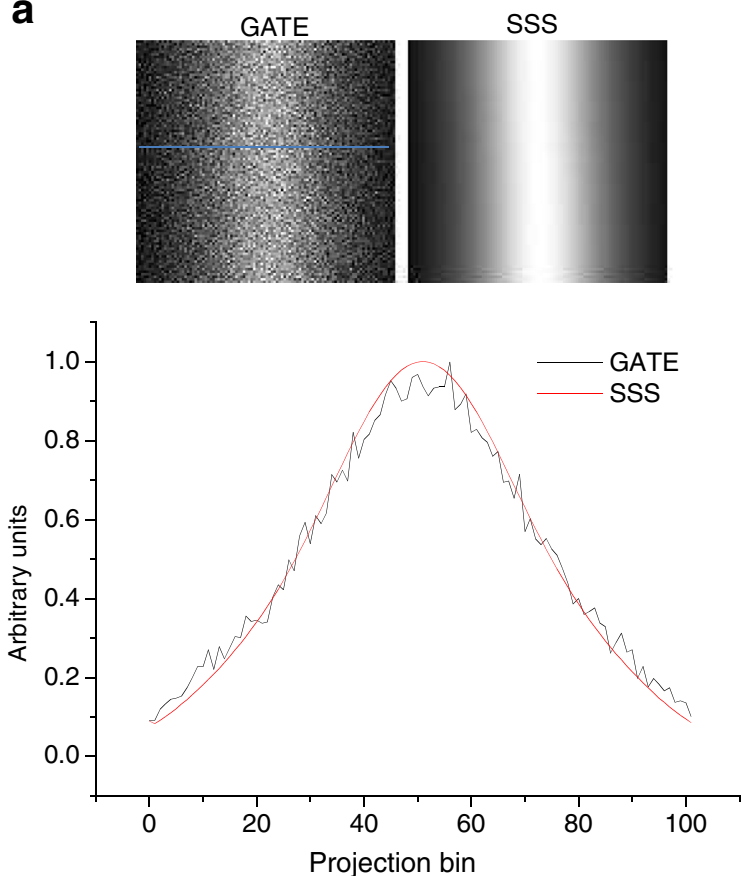

b
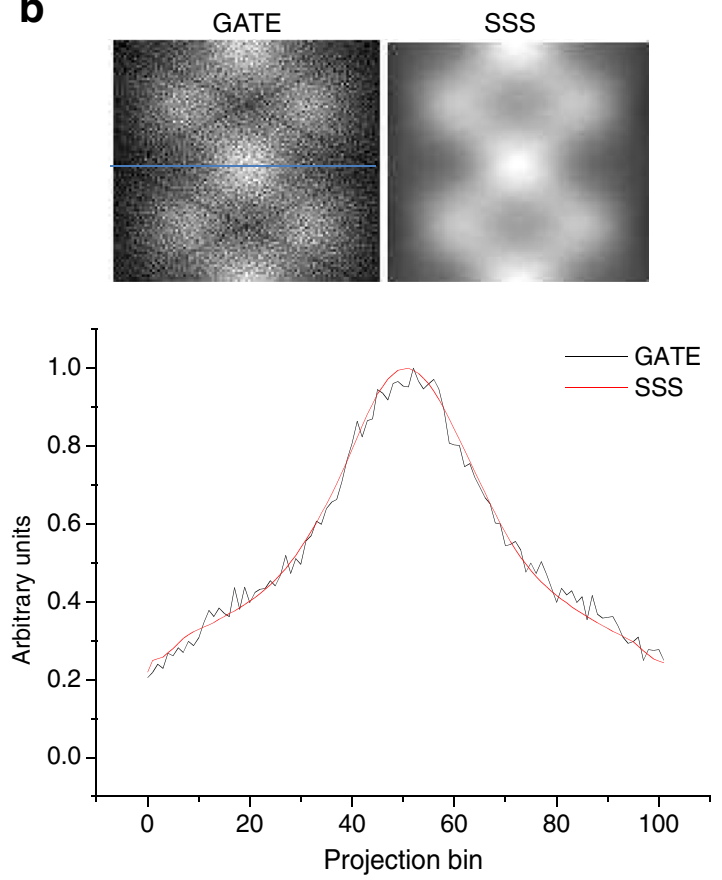

Fig. 3. Comparison between Monte Carlo simulated and model-based scatter sinogram plane (upper row) and corresponding profiles (lower row) for a single and $\mathbf{b}$ multiple five NEMA image quality phantoms. 
a
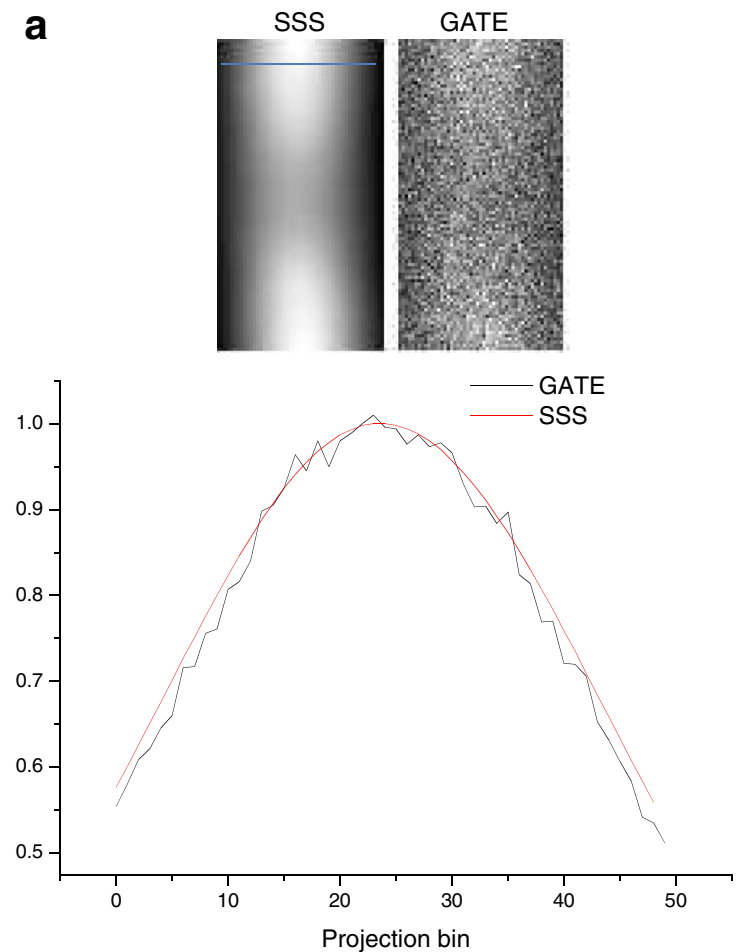

b
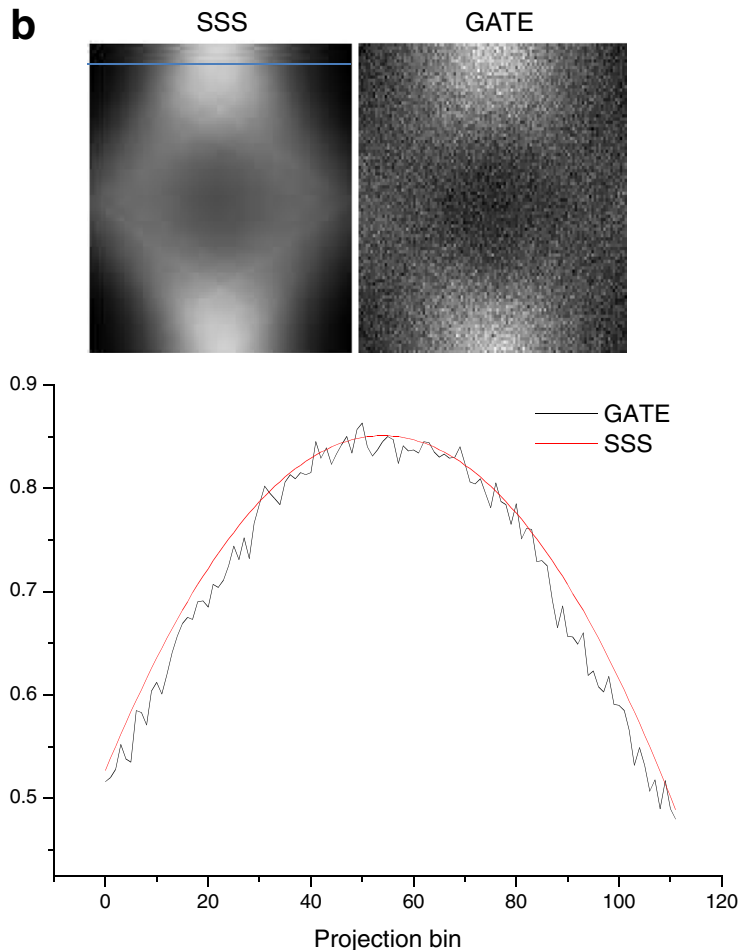

Fig. 4. Comparison of Monte Carlo simulated and model-based scatter component sinogram plane (upper row) and corresponding profiles (lower row) for a single ROBY and $\mathbf{b}$ two ROBY models.

subjects' arrangement. Attenuation correction had a negligible effect on the contrast. However, scatter correction along with attenuation correction improved the contrast for the cold air compartment in single- and multiple-subject PET studies. The impact of scatter correction was more pronounced in simultaneous multiple subjects.

A representative transaxial slice of the NEMA image quality phantom corresponding to single-, three- and fivesubject configurations is shown in Fig. 5. Horizontal profiles through the centre of images corresponding to the true image (unscattered events only) serving as reference, the image reconstructed after attenuation correction only and after applying attenuation and scatter corrections are also shown. It can be seen that scatter correction improves the contrast in the cold cylinders and matches better the true image, especially in the five-subject configuration.
The recovery coefficients in the lung, liver, left and right kidney regions of the MOBY phantom are summarized in Table 5 for the different scanning configurations. The use of attenuation correction overestimates the recovery coefficients especially in the five-subject configuration, whereas attenuation and scatter correction underestimate the recovery coefficients. Higher recovery coefficients are obtained for single compared to multiple-subject imaging after scatter correction. A transverse slice of the MOBY phantom corresponding to single-, three- and five-subject configurations through the kidney region is shown in Fig. 6. It can be seen that scatter correction has little value especially for the single-subject configuration.

The recovery coefficients in the lung, heart and kidney regions of the ROBY phantom reconstructed with attenuation correction only and with attenuation and scatter corrections for

Table 3. Spill-over ratio (SOR) measured in the cold compartments of the NEMA phantom reconstructed without correction (NC), with attenuation correction only (AC) and with attenuation and scatter corrections $(\mathrm{AC}+\mathrm{SC})$ for different scanning configurations. Estimates of the same metric in the actual images are also shown

\begin{tabular}{|c|c|c|c|c|c|c|c|c|c|}
\hline \multirow[t]{2}{*}{ Number of objects } & \multirow[t]{2}{*}{ Position } & \multicolumn{2}{|l|}{ True } & \multicolumn{2}{|l|}{$\mathrm{NC}$} & \multicolumn{2}{|l|}{$\mathrm{AC}$} & \multicolumn{2}{|c|}{$\mathrm{AC}+\mathrm{SC}$} \\
\hline & & Air & Water & Air & Water & Air & Water & Air & Water \\
\hline 1 & Centre & 0.04 & 0.32 & 0.15 & 0.41 & 0.07 & 0.34 & 0.05 & 0.32 \\
\hline \multirow[t]{3}{*}{3} & Centre & 0.06 & 0.42 & 0.17 & 0.43 & 0.10 & 0.48 & 0.07 & 0.40 \\
\hline & Right & 0.05 & 0.38 & 0.15 & 0.45 & 0.09 & 0.51 & 0.06 & 0.39 \\
\hline & Left & 0.06 & 0.41 & 0.14 & 0.46 & 0.11 & 0.53 & 0.08 & 0.40 \\
\hline \multirow[t]{5}{*}{5} & Centre & 0.06 & 0.51 & 0.19 & 0.55 & 0.08 & 0.59 & 0.06 & 0.52 \\
\hline & Right & 0.05 & 0.46 & 0.17 & 0.56 & 0.08 & 0.46 & 0.03 & 0.47 \\
\hline & Left & 0.03 & 0.39 & 0.16 & 0.47 & 0.07 & 0.59 & 0.05 & 0.42 \\
\hline & Up & 0.07 & 0.50 & 0.16 & 0.51 & 0.09 & 0.58 & 0.05 & 0.52 \\
\hline & Down & 0.06 & 0.47 & 0.15 & 0.53 & 0.11 & 0.55 & 0.04 & 0.46 \\
\hline
\end{tabular}


Table 4. Contrast measured in the NEMA phantom reconstructed without correction (NC), with attenuation correction only (AC) and with attenuation and scatter correction $(\mathrm{AC}+\mathrm{SC})$ for different scanning configurations. Values of the same metric in the true images are also shown

\begin{tabular}{|c|c|c|c|c|c|c|c|c|c|}
\hline \multirow[t]{2}{*}{ Number of objects } & \multirow[t]{2}{*}{ Position } & \multicolumn{2}{|l|}{ True } & \multicolumn{2}{|l|}{$\mathrm{NC}$} & \multicolumn{2}{|l|}{$\mathrm{AC}$} & \multicolumn{2}{|c|}{$\mathrm{AC}+\mathrm{SC}$} \\
\hline & & Air & Water & Air & Water & Air & Water & Air & Water \\
\hline 1 & Centre & 71.08 & 52.27 & 61.67 & 48.76 & 62.98 & 49.98 & 70.32 & 51.84 \\
\hline \multirow[t]{3}{*}{3} & Centre & 82.35 & 52.86 & 60.74 & 46.49 & 61.96 & 47.92 & 80.39 & 51.09 \\
\hline & Right & 78.04 & 48.34 & 56.10 & 40.31 & 57.00 & 41.57 & 76.94 & 46.65 \\
\hline & Left & 78.03 & 47.05 & 58.69 & 40.87 & 59.51 & 41.87 & 77.62 & 45.96 \\
\hline \multirow[t]{5}{*}{5} & Centre & 83.68 & 49.44 & 55.63 & 45.08 & 56.99 & 46.04 & 86.66 & 51.33 \\
\hline & Right & 80.31 & 40.41 & 50.45 & 36.09 & 51.24 & 36.63 & 83.36 & 42.37 \\
\hline & Left & 80.63 & 42.75 & 54.63 & 36.04 & 55.34 & 36.48 & 82.05 & 43.41 \\
\hline & $\mathrm{Up}$ & 82.29 & 42.88 & 53.83 & 38.40 & 54.81 & 39.12 & 84.51 & 43.69 \\
\hline & Down & 79.47 & 41.08 & 52.66 & 35.70 & 53.53 & 36.01 & 81.46 & 42.53 \\
\hline
\end{tabular}

single- and two-subject scanning configurations are represented in Fig. 7. The use of scatter correction compensates for the overestimation of recovery coefficients resulting from attenuation correction alone but induces equivalent underestimation. Transaxial slices and corresponding horizontal profiles of the ROBY thorax region representing the true, uncorrected, attenuation corrected only and attenuation and scatter-corrected images are shown in Fig. 8.

\section{Discussion}

In this work, we assessed the magnitude and spatial distribution of the scatter component in small-animal PET when imaging single- and multiple-subject simultaneously.
The impact of scatter correction on image quality and quantitative accuracy under the same conditions was also evaluated. Monte Carlo calculations are widely used to study the characteristics of Compton scattering in PET [35]. Moreover, the Monte Carlo method offers the unique capability to provide detailed information about unscattered and scattered events and even to distinguish between single- and multiplescattered events. Recent developments in computationally efficient Monte Carlo simulation packages combined with advances in computational platforms including graphical processing units and cloud computing has made it possible to model the scatter component in small-animal PET imaging using realistic source distributions within complex anatomical models under controlled conditions [25]. a
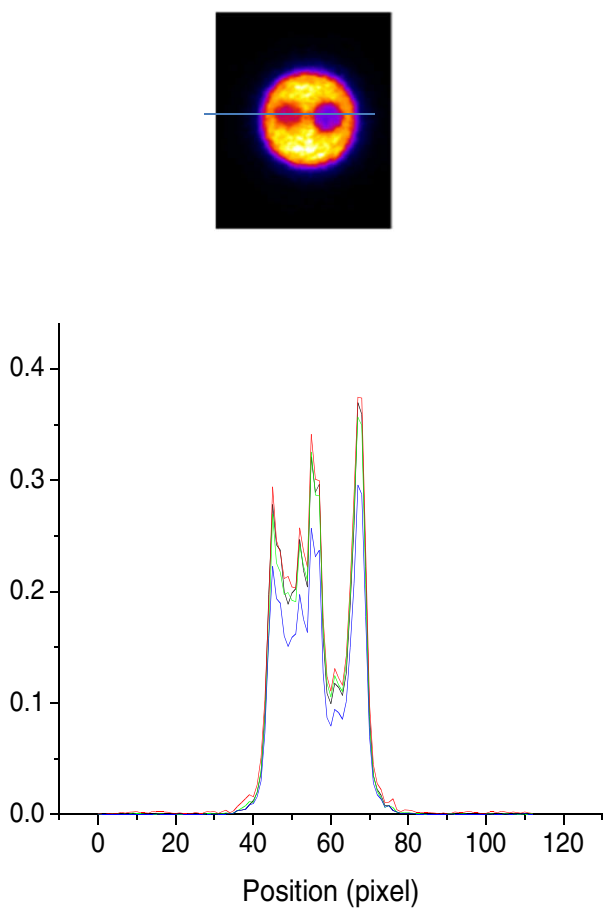

b
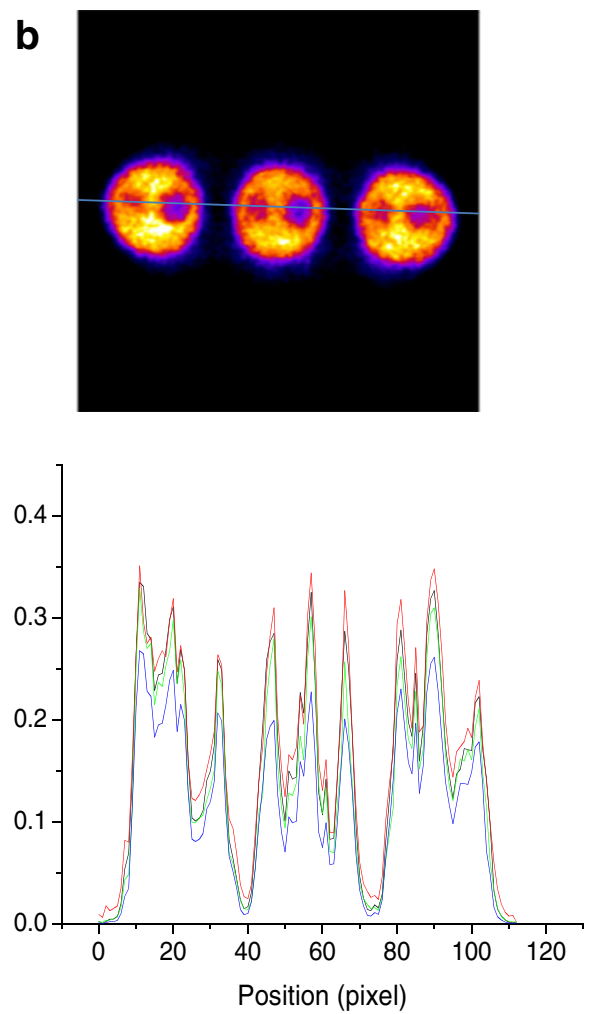

C
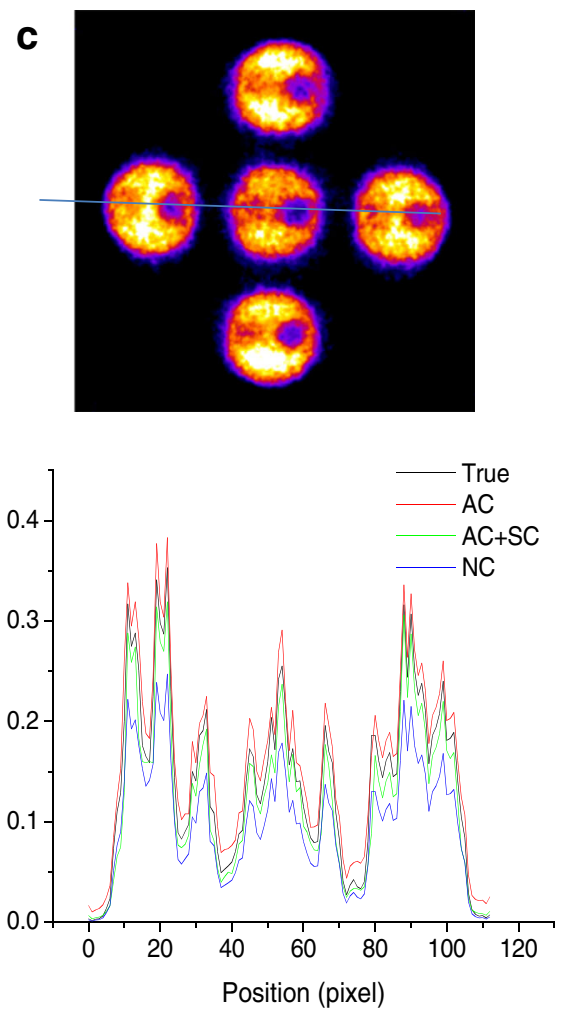

Fig. 5. Reconstructed images of the simulated NEMA image quality phantom (upper row) and their corresponding horizontal profiles (lower row) comparing true, attenuation corrected $(A C)$ and attenuation and scatter-corrected $(A C+S C)$ images for a single, $\mathbf{b}$ three and $\mathbf{c}$ five phantom configurations. The displayed transverse slices correspond to $A C+S C$ images. 
Table 5. Recovery coefficients measured in different body regions of the MOBY phantom reconstructed with attenuation correction only (AC) and with attenuation and scatter corrections $(\mathrm{AC}+\mathrm{SC})$ for different scanning configurations

\begin{tabular}{|c|c|c|c|c|c|c|c|c|c|}
\hline \multirow[t]{2}{*}{ Number of objects } & \multirow[t]{2}{*}{ Position } & \multicolumn{2}{|l|}{ Lung } & \multicolumn{2}{|l|}{ Liver } & \multicolumn{2}{|c|}{ Left kidney } & \multicolumn{2}{|c|}{ Right kidney } \\
\hline & & $\mathrm{AC}$ & $\mathrm{AC}+\mathrm{SC}$ & $\mathrm{AC}$ & $\mathrm{AC}+\mathrm{SC}$ & $\mathrm{AC}$ & $\mathrm{AC}+\mathrm{SC}$ & $\mathrm{AC}$ & $\mathrm{AC}+\mathrm{SC}$ \\
\hline 1 & Centre & 101.66 & 98.35 & 100.81 & 98.34 & 100.20 & 99.00 & 100.55 & 99.03 \\
\hline \multirow[t]{3}{*}{3} & Centre & 100.21 & 93.71 & 100.91 & 95.19 & 100.14 & 95.07 & 99.82 & 94.82 \\
\hline & Right & 99.86 & 95.26 & 99.75 & 94.92 & 99.73 & 95.41 & 100.61 & 97.28 \\
\hline & Left & 99.74 & 92.99 & 100.02 & 94.79 & 100.37 & 96.74 & 100.26 & 96.18 \\
\hline \multirow[t]{5}{*}{5} & Centre & 104.47 & 93.06 & 105.12 & 92.66 & 100.84 & 90.62 & 102.26 & 91.10 \\
\hline & Right & 102.11 & 93.32 & 102.98 & 92.72 & 99.45 & 91.37 & 100.78 & 93.13 \\
\hline & Left & 105.84 & 95.48 & 105.87 & 96.32 & 100.20 & 93.38 & 101.69 & 92.20 \\
\hline & Up & 103.86 & 96.38 & 101.87 & 93.66 & 100.38 & 94.20 & 102.16 & 95.53 \\
\hline & Down & 101.78 & 93.77 & 103.59 & 96.03 & 102.77 & 97.19 & 101.39 & 94.76 \\
\hline
\end{tabular}

In this work, the GATE platform was used to study the scatter characteristics of the LabPET-8 scanner. It was observed that the scatter contribution from kovar covering material was significantly higher in comparison to scatter originating from lead and copper plate serving as end-of-FOV shielding material. This is due to the fact that kovar, being a high-density alloy covering the entire detectors, produces more Compton scattering in the LabPET-8 scanner. Similar observations regarding the behaviour of this material were reported more recently [18]. In particular, when imaging rats, organs presenting with high uptake such as the heart, brain and bladder can be outside the imaging FOV owing to the small axial FOV of the scanner. This might increase detected scatter originating from outside the FOV. Therefore, the contribution of scattered events arising from outside the FOV was evaluated. It was observed that events arising from outside the FOV have a negligible impact $(<1 \%)$ for LETs of 350 and $450 \mathrm{keV}$, whereas the contribution was less than $1.7 \%$ for a LET of $250 \mathrm{keV}$, thus indicating that the end shields of the LabPET- 8 scanner are reasonably effective in rejecting out-of-FOV scatter. This also implies that a scatter correction method that models only scattered events originating within the FOV, such as the single-scatter simulation method used in this study [33], is reasonably adequate is small-animal PET imaging. The object SF was evaluated for single and multiple scanning of the NEMA image quality phantom, MOBY and different body regions of the ROBY anatomical model. The SF increases substantially for simultaneous multiple subjects imaging compared to single-subject imaging, implying that scatter correction is important when imaging simultaneous multiple subjects. a

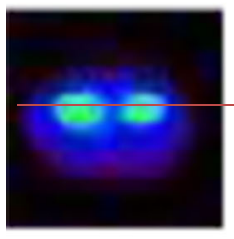

b

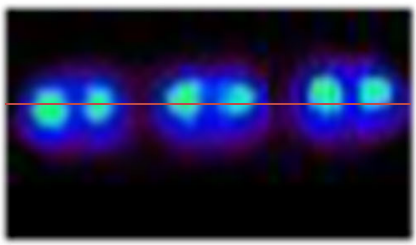

C
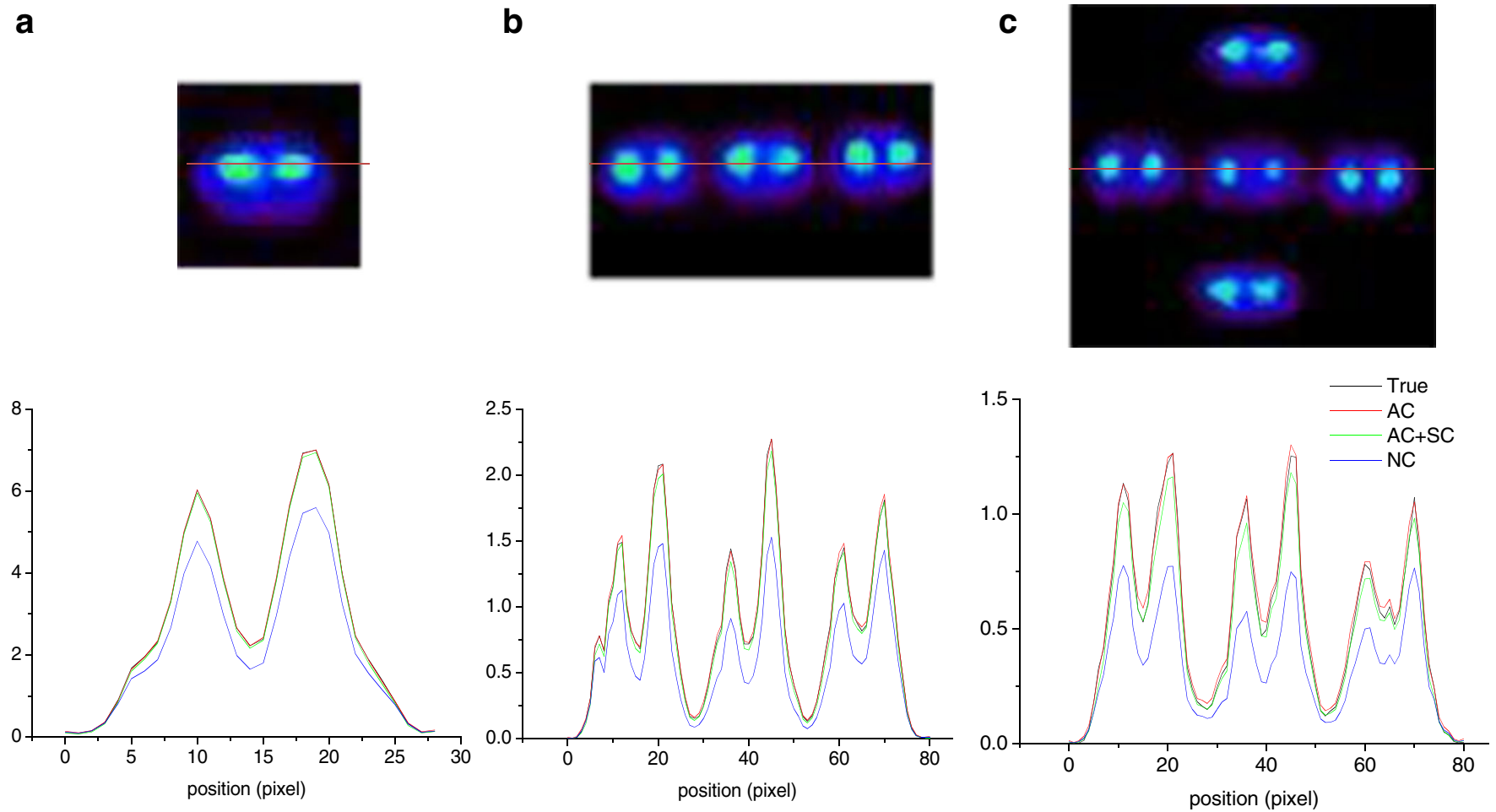

Fig. 6. Representative slices of the simulated MOBY phantom (upper row) and their corresponding horizontal profiles (lower row) comparing true, uncorrected $(N C)$, attenuation corrected $(A C)$ and attenuation and scatter-corrected $(A C+S C)$ images for a single, $\mathbf{b}$ three and $\mathbf{c}$ five MOBY model configurations. 

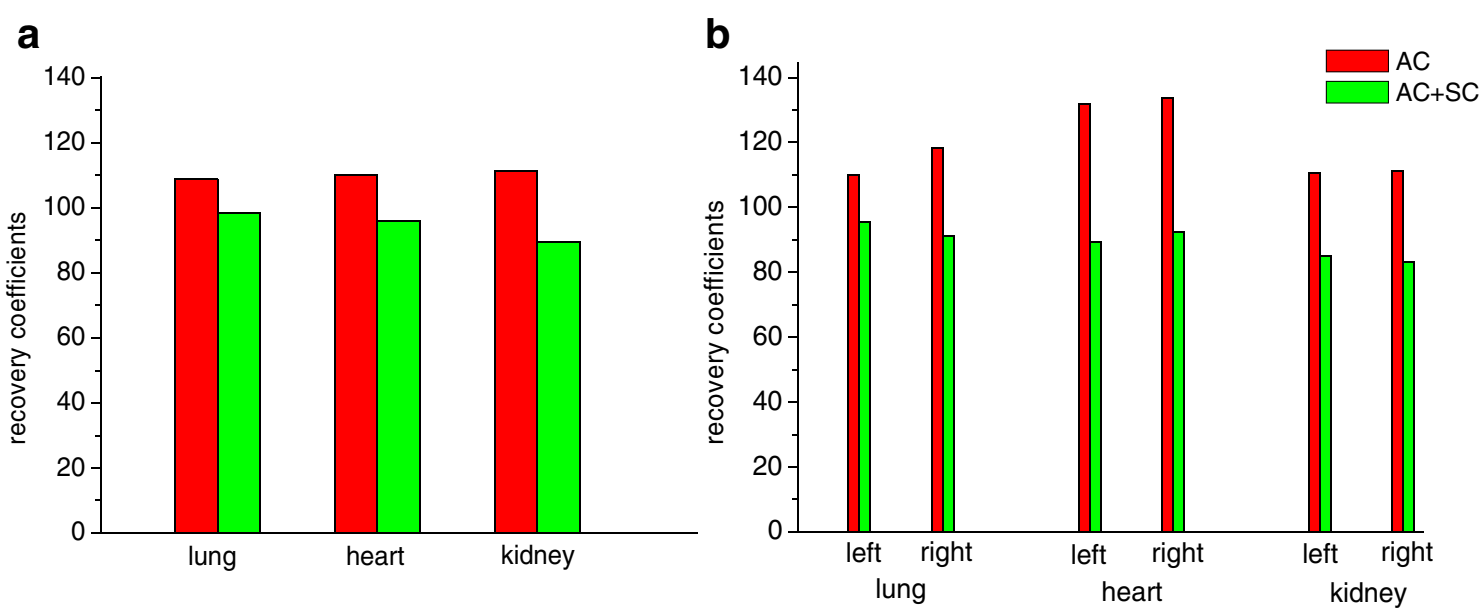

Fig. 7. Recovery coefficients measured in different body regions of the ROBY phantom reconstructed with attenuation correction only $(A C)$ and with attenuation and scatter correction $(A C+S C)$ for $\mathbf{a}$ single and $\mathbf{b}$ two ROBY models.

Since APDs are inherently slow, APD-based systems such as the LabPET-8 scanner require a wide coincidence window as well as a wide energy window. Also, different timing windows are used for LGSO-LGSO, LYSO-LYSO and LGSO-LYSO in LabPET-8 scanner. Hence, the SF was evaluated under realistic conditions for a selected coincidence a

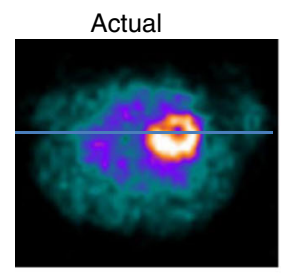

$\mathrm{AC}$

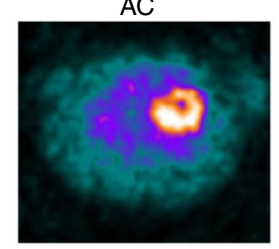

b
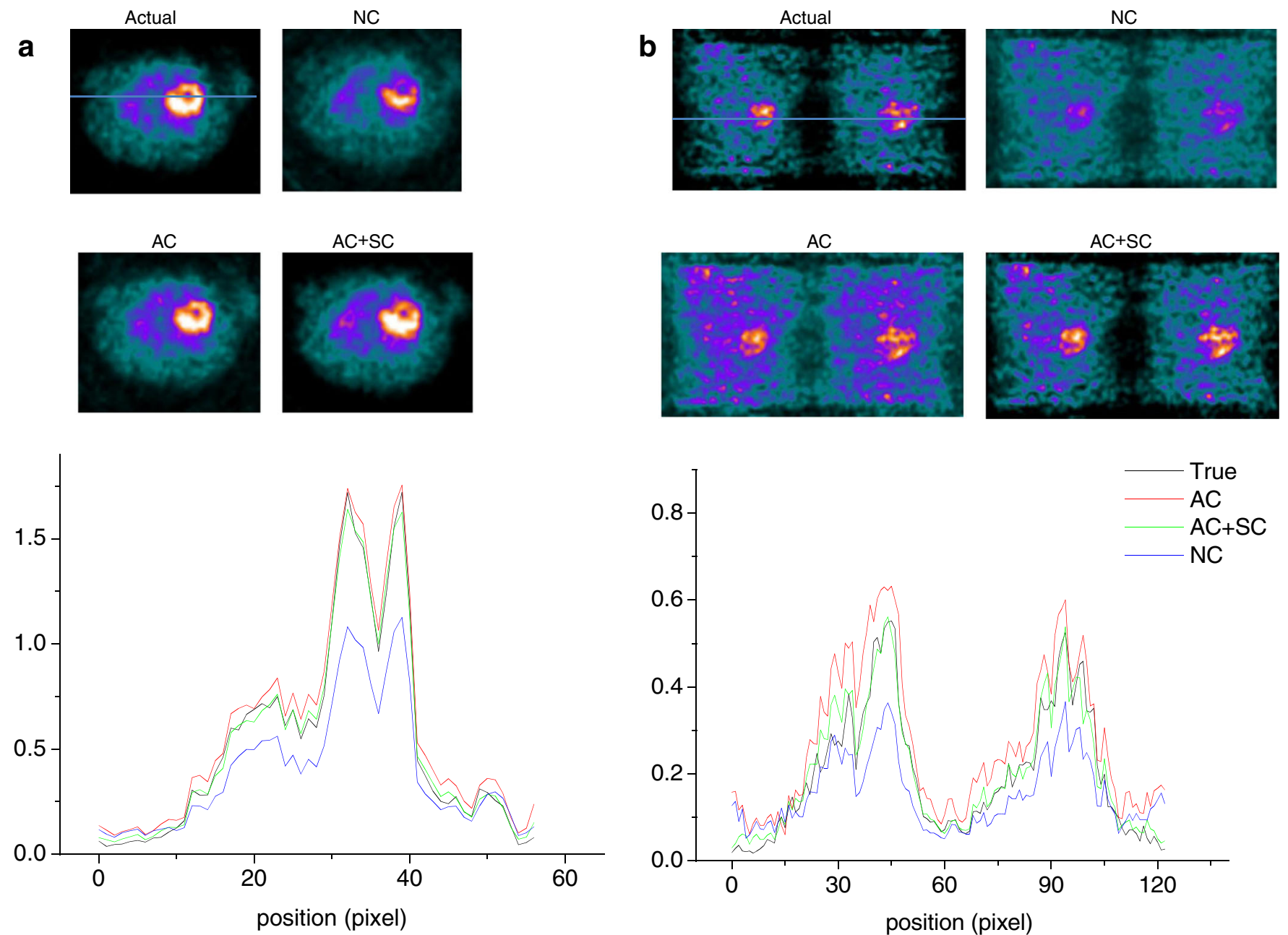

$\mathrm{AC}$
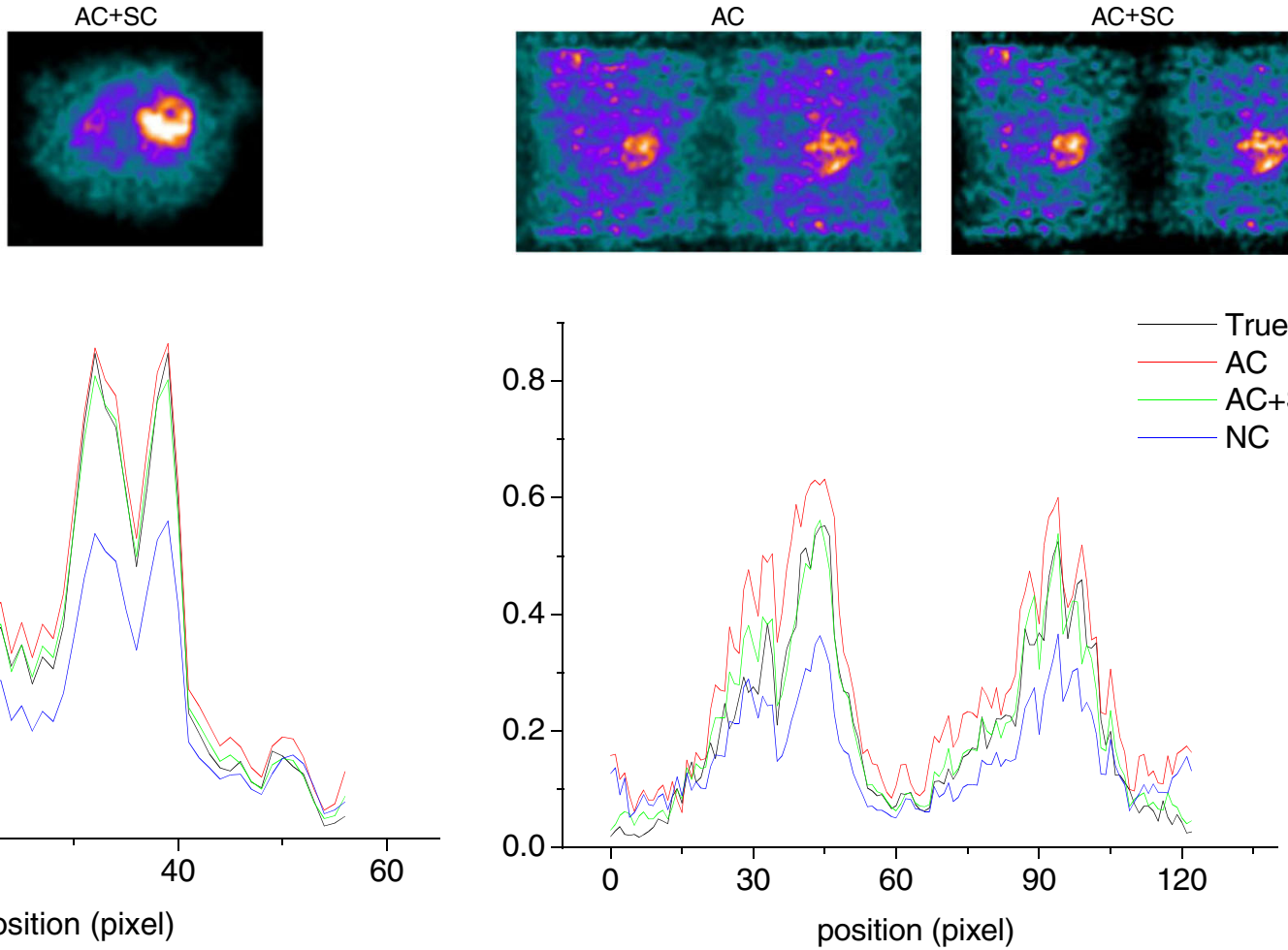

Fig. 8. Representative slices of the simulated ROBY thorax region (upper row) and their corresponding horizontal profiles (lower row) comparing true, uncorrected (NC), attenuation corrected $(A C)$ and attenuation and scatter-corrected $(A C+S C$ ) for a single and $\mathbf{b}$ two ROBY models. 
timing window of $10 \mathrm{~ns}$ and lower energy threshold of 250,350 and $425 \mathrm{keV}$ (the upper threshold was set to $650 \mathrm{keV}$ ). As expected, the SF decreased with increasing the LET [8].

The estimated scatter distribution using the single-scatter simulation method shows good agreement with Monte Carlo simulations for single- and simultaneous multiple-subject imaging. The SNR for scatter-corrected images deteriorates owing to the scatter subtraction process and the consequent reduction of statistics in the acquired data sets. However, the quantitative accuracy is improved especially for simultaneous multiple-subject imaging. The spill-over ratio increases with increasing the number of subjects in the FOV. Scatter correction improves the SOR for both cold water and air compartments in single- and multiple-subject imaging; however, this effect is much noticeable for the air compartment. Similar observations about the SOR were reported in a related study [21]. The model-based scatter correction technique improves image contrast for both cold compartments of the NEMA image quality phantom compared to the case where no correction is performed. Likewise, the recovery coefficients in different body regions of the MOBY and ROBY phantoms were remarkably improved after scatter correction for simultaneous multiple mice as well as single and multiple rat imaging.

\section{Conclusion}

The scatter component originating from different parts of the LabPET-8 scanner including gantry and out-of field-of-view was characterized. The magnitude and spatial distribution of Compton scattering were evaluated for single and simultaneous multiple subjects. Model-based estimation of the scatter component has good agreement with corresponding Monte Carlo simulations. Attenuation and scatter corrections improve contrast and quantification in small-animal PET especially for simultaneous multiple-subject imaging. For accurate PET quantification, attenuation correction is required for single and multiple mice and rat studies, whereas scatter correction is required for single rat and multiple mice and rat studies.

Acknowledgments. This work was supported by the Swiss National Science Foundation under grant SNSF 3152A0-102143, Geneva Cancer League and the Indo-Swiss Joint Research Programme ISJRP 138866. The authors would like to thank Jean-Daniel Leroux and Roger Lecomte from the University of Sherbrooke, Canada, for their help with Monte Carlo modelling of the LabPETTM scanner.

Conflict of Interest. The authors declare that they have no conflict of interest.

\section{References}

1. Levin CS, Zaidi H (2007) Current trends in preclinical PET system design. PET Clinics 2:125-160

2. Rowland DJ, Cherry SR (2008) Small-animal preclinical nuclear medicine instrumentation and methodology. Sem Nucl Med 38:209-222

3. Visser EP, Disselhorst JA, van Lier MGJTB et al (2011) Characterization and optimization of image quality as a function of reconstruction algorithms and parameter settings in a Siemens Inveon small-animal
PET scanner using the NEMA NU 4-2008 standards. Nucl Instrum Meth A 629:357-367

4. Dupont P, Warwick J (2009) Kinetic modelling in small animal imaging with PET. Methods 48:98-103

5. Vaska P, Rubins DJ, Alexoff DL, Schiffer WK (2006) Quantitative imaging with the micro-PET small-animal PET tomograph. Int Rev Neurobiol 73:191-218

6. Fahey FH, Gage HD, Buchheimer N et al (2004) Evaluation of the quantitative capability of a high-resolution positron emission tomography scanner for small animal imaging. J Comput Assist Tomogr 28:842-848

7. Mannheim JG, Judenhofer MS, Schmid A et al (2012) Quantification accuracy and partial volume effect in dependence of the attenuation correction of a state-of-the-art small animal PET scanner. Phys Med Biol 57:3981-3993

8. Prasad R, Zaidi H (2012) A cone-shaped phantom for assessment of small animal PET scatter fraction and count rate performance. Mol Imaging Biol 14:561-571

9. Zaidi H, Koral KF (2004) Scatter modelling and compensation in emission tomography. Eur J Nucl Med Mol Imaging 31:761-782

10. Schöder H, Erdi Y, Larson S, Yeung HD (2003) PET/CT: a new imaging technology in nuclear medicine. Eur J Nucl Med Mol Imaging 30:1419-1437

11. Yang Y, Cherry SR (2006) Observations regarding scatter fraction and NEC measurements for small animal PET. IEEE Trans Nucl Sci 53:127-132

12. Konik A, Koesters T, Madsen MT, Sunderland JJ (2011) Evaluation of attenuation and scatter correction requirements as a function of object size in small animal PET imaging. IEEE Trans Nucl Sci 58:2308-2314

13. Prasad R, Ay MR, Ratib O, Zaidi H (2011) CT-based attenuation correction on the FLEX Triumph ${ }^{\mathrm{TM}}$ preclinical PET/CT scanner. IEEE Trans Nucl Sci 58:66-75

14. Bao Q, Newport D, Chen M, Stout DB, Chatziioannou AF (2009) Performance evaluation of the Inveon dedicated PET preclinical tomograph based on the NEMA NU-4 standards. J Nucl Med 50:401408

15. Huisman MC, Reder S, Weber AW et al (2007) Performance evaluation of the Philips MOSAIC small animal PET scanner. Eur J Nucl Med Mol Imaging 34:532-540

16. Prasad R, Ratib O, Zaidi H (2010) Performance evaluation of the FLEX Triumph $^{\mathrm{TM}}$ X-PET scanner using the NEMA NU-04 standards. J Nucl Med 51:1608-1615

17. Prasad R, Ratib O, Zaidi H (2011) NEMA NU-04-based performance characteristics of the LabPET- $8^{\mathrm{TM}}$ small animal PET scanner. Phys Med Biol 56:6649-6664

18. Goertzen AL, Bao Q, Bergeron M et al (2012) NEMA NU 4-2008 comparison of preclinical PET imaging systems. J Nucl Med 53:13001309

19. Alexoff DL, Vaska P, Marsteller D et al (2003) Reproducibility of 11Craclopride binding in the rat brain measured with the microPET R4: effects of scatter correction and tracer specific activity. J Nucl Med 44:815-822

20. Aide N, Desmonts C, Briand M et al (2010) High-throughput small animal PET imaging in cancer research: evaluation of the capability of the Inveon scanner to image four mice simultaneously. Nucl Med Commun 31:851-858

21. Siepel FJ, van Lier MGJTB, Chen M, Disselhorst JA, Meeuwis APW, Oyen WJG et al (2010) Scanning multiple mice in a small-animal PET scanner: influence on image quality. Nucl Instr Meth A 621:605-610

22. Aide N, Visser EP, Lheureux $\mathrm{S}$ et al (2012) The motivations and methodology for high-throughput PET imaging of small animals in cancer research. Eur J Nucl Med Mol Imaging 39:1497-1509

23. Habte F, Ren G, Doyle T, et al (2011) High-throughput multiple mice imaging on microPET and microPET-CT scanners: Evaluation on image quantitation effect [abstract]. Proceedings of the World Molecular Imaging Congress, p. P568.

24. Bergeron M, Cadorette J, Beaudoin JF, Lepage MD, Robert G, Selivanov V et al (2009) Performance evaluation of the LabPET APD-based digital PET scanner. IEEE Trans Nucl Sci 56:10-16

25. Zaidi H (1999) Relevance of accurate Monte Carlo modeling in nuclear medical imaging. Med Phys 26:574-608

26. Jan S, Santin G, Strul D et al (2004) GATE: a simulation toolkit for PET and SPECT. Phys Med Biol 49:4543-4561 
27. Thielemans K, Tsoumpas C, Mustafovic S et al (2012) STIR: software for tomographic image reconstruction release 2. Phys Med Biol 57:867883

28. Zaidi H, Xu XG (2007) Computational anthropomorphic models of the human anatomy: the path to realistic Monte Carlo modeling in medical imaging. Annu Rev Biomed Eng 9:471-500

29. Kesner AL, Dahlbom M, Huang SC et al (2006) Semiautomated analysis of small-animal PET data. J Nucl Med 47:1181-1186

30. Gutierrez DF, Zaidi H (2012) Automated analysis of small animal PET studies through deformable registration to an atlas. Eur J Nucl Med Mol Imaging 39:1807-1820

31. National Electrical Manufacturers Association (2008) NEMA Standards Publication NU 4-2008. Performance Measurements of Small Animal
Positron Emission Tomographs. Rosslyn, VA: National Electrical Manufacturers Association.

32. Segars WP, Tsui BM, Frey EC et al (2004) Development of a 4-D digital mouse phantom for molecular imaging research. Mol Imaging Biol 6:149-159

33. Watson CC (2000) New, faster, image-based scatter correction for 3D PET. IEEE Trans Nucl Sci 47:1587-1594

34. Tsoumpas C, Aguiar P, Nikita KS et al (2004) Evaluation of the single scatter simulation algorithm implemented in the STIR library. IEEE Nucl Sci Symp Conf Rec 6:3361-3365

35. Adam LE, Karp JS, Brix G (1999) Investigation of scattered radiation in 3D whole-body positron emission tomography using Monte Carlo simulations. Phys Med Biol 44:2879-2895 